\title{
Data sequences and subjective sampling distributions'
}

LEE ROY BEACH AND JON B. OLSON THE UNIVERSITY OF WASHINGTON

Changes in subjective sampling distributions as a function of the structure of observed data sequences were examined as an explanation of errors in subjective probability revisions. Sequences influenced the distributions and this in turn was reflected in revision.

A procedure often used to study subjective processing of probabilistic information starts by $\mathrm{E}$ showing $\mathrm{S}$ two urns, one containing, say, .60 red balls and .40 green balls, and the other containing .40 red balls and .60 green balls. Then $E$ flips a coin to select one of the urns, samples from the urn, and shows the ball (datum) to S. S's task is to revise his initial subjective probabilities (SPs) of .50-.50 about which urn was selected in light of the information provided by the ball. The ball is replaced, another drawn from the same urn, $S$ revises his previous revision, etc., for a number of draws.

When S's revisions are compared to the normative revisions derived from Bayes' theorem it is found that they often are systematically inaccurate (e.g., Phillips \& Edwards, 1966). One theoretical explanation of the error is that Ss incorrectly interpret the implications of the observed datum, although they correctly utilize their incorrect interpretation in revising their SPS about the urns. Formally, this is the same as saying that $\mathrm{Ss}$ behave as Bayes' theorem prescribes but that their subjective sampling distributions (SSDs) are inaccurate (Peterson, DuCharme, \& Edwards, 1967; Wheeler \& Beach, 1967). However, examination of individual S's data shows that successive revisions for data from the same urn do not always appear to be based on the same SSDs. That is, while use of objective sampling distributions dictates that no matter where it is in the sequence of draws a given datum always has the same implications for the amount of revision to be done, $\mathrm{Ss}^{\prime}$ revisions do not always conform to such a restraint. This is negative evidence for the theory, but a solution is suggested by the results of other kinds of experiments in which Ss see sequences of binary data (e.g., Jarvik, 1951). In these, $\mathrm{Ss}^{\prime}$ behavior apparently is influenced by opinions about the lengths of event runs to be expected from various binomial populations. SPs for the next datum change from trial to trial as a function of runs rather than remaining constant throughout the experiment; in our terms, the SSDs change. The purpose of this experiment was to see if SSDs are influenced by the structure of sequences of draws from the urn and, if so, if these changes are reflected in SP revisions.
Method

The paradigm described above was used with urns of .60 red-.40 green balls and .60 green- .40 red balls (called $U_{R}$ and $U_{G}$, respectively), and with the 30 data sequences listed in Fig. 1. After each sequence Ss made two bets, one about the nextball to be expected if $E$ were sampling $U_{R}$ and a similar bet for $U_{G}$. Then a final ball was drawn and $S s$ made their final revision. Fifteen of the final draws were red and 14 were green. No feedback was given about which urn was being sampled. Ss made revisions by moving markers in two calibrated troughs, one for each urn. After revising, $S$ wrote the two probabilities, constrained to add to 1.00 , on an answer sheet. Successive revisions took up where the last had left the markers; markers were reset to $.50-.50$ before each sequence began. Ss made bets by selecting pairs of payoffs for which they were willing to gamble from lists that are described in detail in Beach \& Phillips (1967). These bets permitted inference of SPs of the next draw being red or green given each of the urns. Objectively the bets should not vary, but for only two of the 24 male college Ss was this true; their data were not used in the analysis.

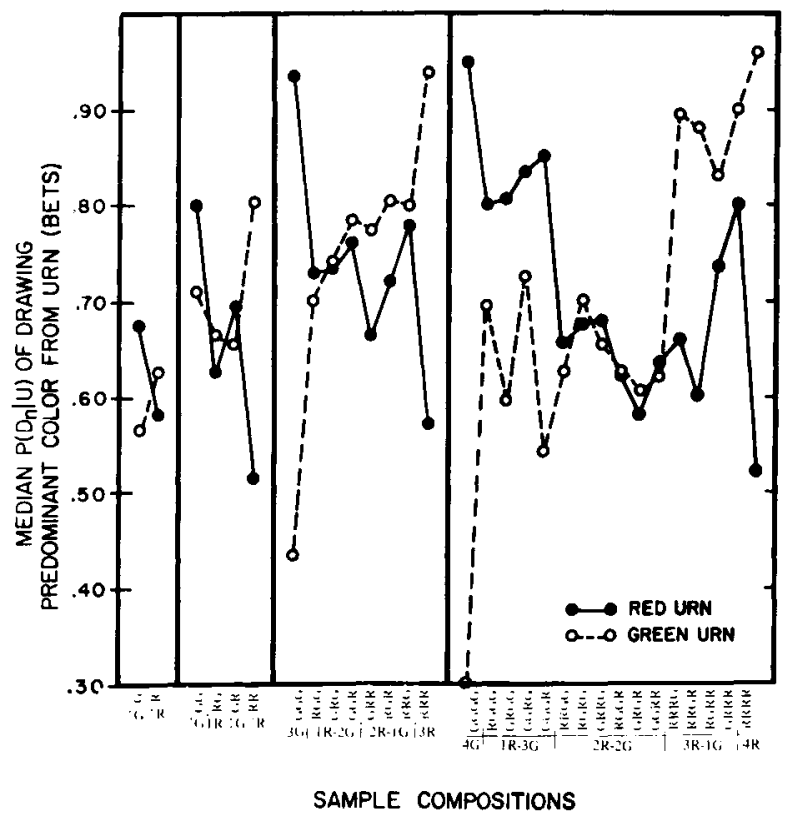

Fig. 1. The effects of draw sequences on the subjective probabilities of the next ball being red or green assuming $U_{R}$ and $U_{G}$ is being sampled. 


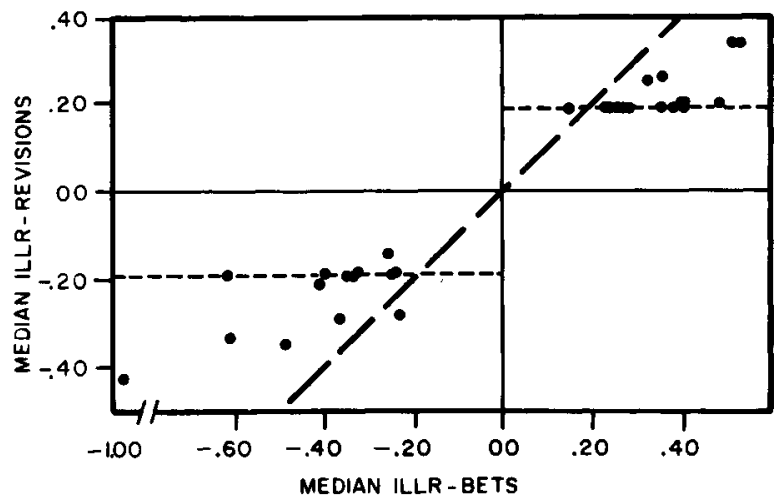

Fig. 2. The relationship between ILLR-Revisions and ILLR-Bets.

\section{Results}

Bayes' theorem, in ratio form, for the last draw is

$$
\frac{P\left(U_{R} \mid D_{n}\right)}{P\left(U_{G} \mid D_{n}\right)}=\frac{P\left(D_{n} \mid U_{R}\right)}{P\left(D_{n} \mid U_{G}\right)} \frac{P\left(U_{R} \mid D_{n-1}\right)}{P\left(U_{G} \mid D_{n-1}\right)}
$$

The probabilities in the ratio on the right are for $U_{R}$ and $U_{G}$ after the revision for the next to the last datum, $D_{n-1}$. The probabilities in the ratio in the middle, the likelihood ratio, are for the last datum, $D_{n}$, having occurred assuming $\mathrm{U}_{\mathrm{R}}$ or $\mathrm{U}_{\mathrm{G}}$ is being sampled; these come from binomial sampling distributions. The probabilities in the ratio on the left are for the two urns after observation of the last datum, $D_{n}$. Because $S$ has recorded his SP for the ratios at either end of the equation, his subjective likelihood ratio can be inferred by subtracting the log of the ratio on the right from that on the left; this will be called the ILLR-Rev. A similar likelihood ratio can be constructed using the SPs inferred from the S's bets. This will be called ILLR-Bets. If the hypothesis is correct, the subjective $P\left(D_{n} \mid U\right)$ from the bets, and the ILLR-Bets constructed from them, should vary as a function of the data sequences. The ILLR-Rev should vary as a function of the ILLR-Bets.

Figure 1 shows how the subjective $P\left(D_{n} \mid U\right)$ change as a function of sequences. There tend to be plateaus for equivalent samples, e.g., all having 1 red-3 green.
This implies that the draw order is not as important as the total number of each kind of ball. However, the SPs for the next draw (the SSDs) are influenced differentially by the latter property of the sequences.

In Fig. 2 median ILLR-Bets are compared with median ILLR-Rev for each of the 30 sequences. ILLRs are symmetric around zero, and those favoring $U_{G}$ are negative. If $\mathrm{Ss}^{\prime}$ revisions were always accurate all points would be on the horizontal dashed lines. If the ILLR-Bets, i.e., the varying SSDs, completely accounted for the revisions, the two kinds of ILLRs would be on the oblique dashed line.

Figure 2 shows four things: (1) There were no offquadrant points; the two kinds of ILLRs always agreed on the urn favored by the last draw. (2) Most revisions were accurate; 19 of the 30 ILLR-Rev were within .02 of the objective value of .176. (3) When the ILLR-Rev were inaccurate they deviated toward agreement with the ILLR-Bets; the Pearson correlation between $a b$ solute values of the median ILLRs is .70 ( $<<.01)$. (4) The deviations of the ILLR-Rev from accuracy is not as great as the deviations of the ILLR-Bets. This suggests that the betting procedure itself may have produced extreme bets that in turn led to extreme ILLR-Bets. Alternatively, Vlek (1966) has suggested that Ss may not always rely on likelihood ratios composed of extreme SPs; this hypothesis is currently being examined in a separate experiment.

\section{References}

BEACH, L. R., \& PHILLIPS, L. D. Subjective probabilities inferred from estimates and bets. J. exp. Psychol, in press.

JARVIK, M. E. Probability learning and a negative recency effect in the serial anticipation of alternative symbols. J. exp. Psychol., 1951, 41, 291-297.

PETERSON, C. R., DuCHARME, \& EDWARDS, W. Sampling distributions and probability revisions. $J$. exp. Psychol., in press.

PHILLIPS, L. D., \& EDWARDS, W. Conservation in a simple probability inference task. J. exp. Psychol, 1966, 72, 346-354.

VLEK, C. The use of probabilistic information in decision making. Psychol. Inst. Rep. No. 009-65, 1965, Univ. of Leiden, Netherlands.

WHEELER, G., \& BEACH, L. R. Subjective sampling distributions and conservatism. Organ. Behav. \& Hum. Perform, in press.

Note

1. Supported by U. S. Public Health Service Grant No. MH 13735-01 from the National Institute of Mental Health. 